Universidad de Lima

Facultad de Psicología

Carrera de Psicología

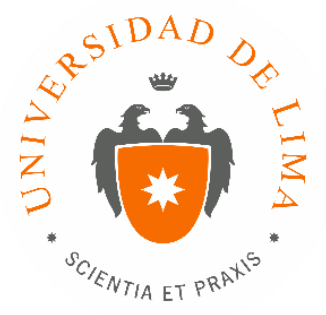

\title{
PLAN DE MEJORA EN EL PROCESO DE RECLUTAMIENTO Y SELECCIÓN PARA UNA SUB ÁREA DE UNA EMPRESA DE SERVICIOS DE AUDITORÍA Y CONSULTORÍA
}

Trabajo de suficiencia profesional para optar el título profesional de Licenciado en Psicología

Karla Patricia Salazar Beronio

Código 20061999

Lima - Perú

Febrero de 2019 


\section{PLAN DE MEJORA EN EL PROCESO DE RECLUTAMIENTO Y SELECCIÓN PARA UNA SUB ÁREA DE UNA EMPRESA DE SERVICIOS DE AUDITORÍA Y CONSULTORÍA}




\section{TABLA DE CONTENIDO}

INTRODUCCIÓN

CAPÍTULO I: IDENTIFICACIÓN DEL PROBLEMA..........................................................9

CAPÍTULO II: DESCRIPCIÓN DE LAS ACTIVIDADES REALIZADAS ....................12

2.1 Elaboración el flujo del proceso de reclutamiento y selección .................................. 12

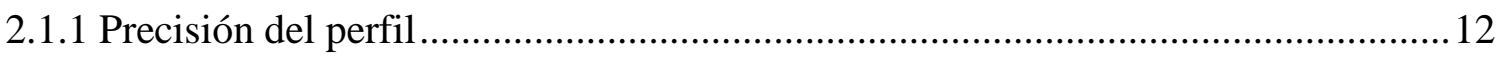

2.1.2 Identificación de la estrategia de reclutamiento y acciones de atracción ................. 13

2.1.3 Establecimiento de las técnicas de evaluación ....................................................... 14

2.1.4 Elaboración de referencias laborales ................................................................... 17

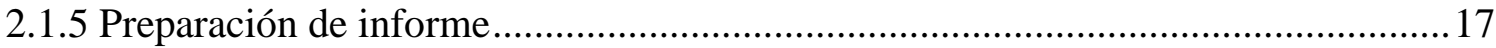

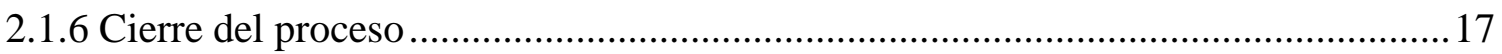

CAPÍTULO III: RESULTADOS DE LA INTERVENCIÓN..................................................18

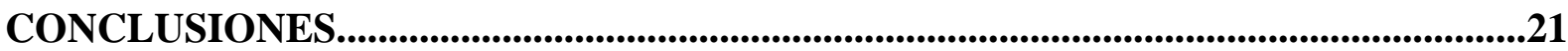

RECOMENDACIONES .......................................................................................................................22

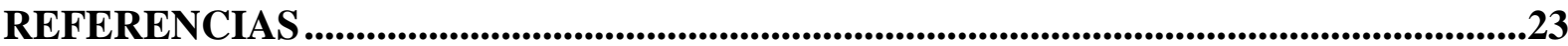




\section{ÍNDICE DE FIGURAS}

Figura 1.1 Variación porcentual del PBI en el periodo 2008-2015 ......................................9

Figura 1.2. Evolución del sistema financiero (en miles de $\mathrm{S} /$ ) .............................................

Figura 1.3. Países con mayor dificultad para cubrir sus vacantes en el 2015 ......................... 11

Figura 3.1. Medios de reclutamiento utilizados.............................................................. 18 


\section{ÍNDICE DE TABLAS}

Tabla 3.1 Resultados de los dos procesos masivos de practicantes..................... 19

Tabla 3.2 Resultados del procesos masivo de practicantes..............................19 


\section{ÍNDICE DE APÉNDICES}

Apéndice 1. Flujo del proceso de reclutamiento y selección..........................25

Apéndice 2. Guía de evaluación para el assessment center............................. .26

Apéndice 3. Cronograma del proceso masivo para la selección de practicantes y asistentes. 27

Apéndice 4. Modelo de informe del candidato...................................... 28

Apéndice 5. Encuesta realizada a los nuevos ingresos por la metodología de Assessment center.... 


\section{INTRODUCCIÓN}

En la actualidad, el proceso de reclutamiento y selección es una de las piezas claves de la dirección del talento, la cual debe tener visión estratégica en el negocio y estar acorde a las exigencias del mundo globalizado, buscando los perfiles que se adecúen a las nuevas necesidades de las organizaciones en un entorno de constantes cambios. Según Salgado y Moscoso (2008) "la selección de personal es un proceso crítico debido a que se toma decisiones sobre el ajuste de los candidatos a los puestos ofrecido, por lo que si se realiza de forma correcta permitirá la incorporación de efectivos de alto rendimiento que influirán en el éxito de la organización" (p. 17).

En el primer capítulo del presente trabajo, se centra en la problemática del proceso de reclutamiento y selección de la sub área de una importante empresa multinacional de servicios de auditoría y consultoría. En consecuencia, se identifica la necesidad de implementar un plan de mejora para una mayor efectividad del proceso.

En el segundo capítulo, se detallan las principales actividades realizadas en el plan de mejora donde se establece la definición del flujo del proceso, acciones de reclutamiento y técnicas de evaluación acorde a los perfiles que se buscaban.

En el tercer capítulo se describen los resultados obtenidos de la implementación de las mejoras en el proceso de reclutamiento y selección para practicantes y asistentes.

Por último, se indican las conclusiones obtenidas de las actividades realizadas y recomendaciones para futuras investigaciones del tema. 


\section{CAPÍTULO I: IDENTIFICACIÓN DEL PROBLEMA}

En el Perú, se ha originado un constante crecimiento de la economía en la última década según se puede apreciar en la figura 1, por lo que se produce un aumento de oferta laboral por satisfacer (Instituto Nacional de Estadística e Informática, 2018).

Figura 1.1

Variación porcentual del PBI en el periodo 2008-2015.

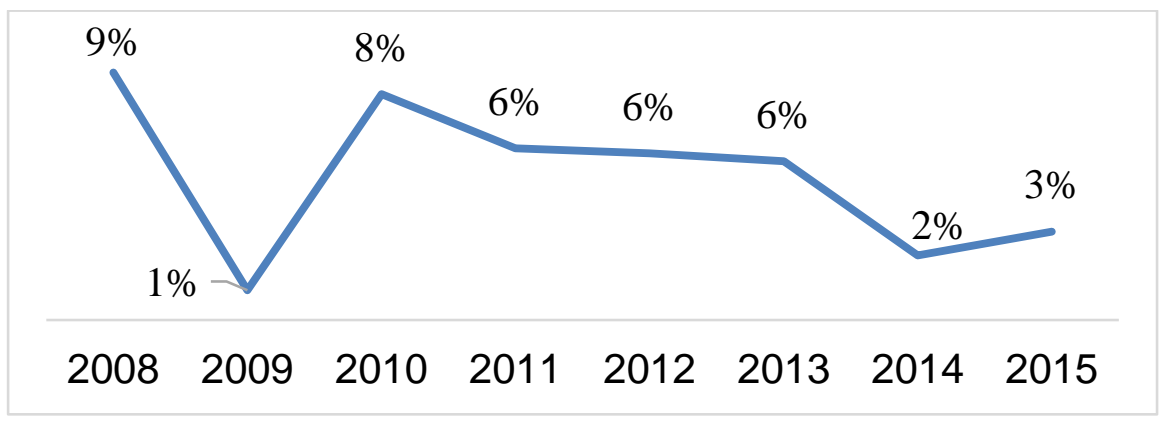

Fuente: Instituto Nacional de Estadística e Informática (2018). Elaboración propia

Dentro de este crecimiento, el sector de la industria financiera ha tenido un papel principal. Como se puede apreciar en la figura 2, ha tenido un desarrollo sostenido en los últimos nueve años (Ey Perú, 2016).

Figura 1.2.

Evolución del sistema financiero (en miles de $\mathrm{S} /$ )

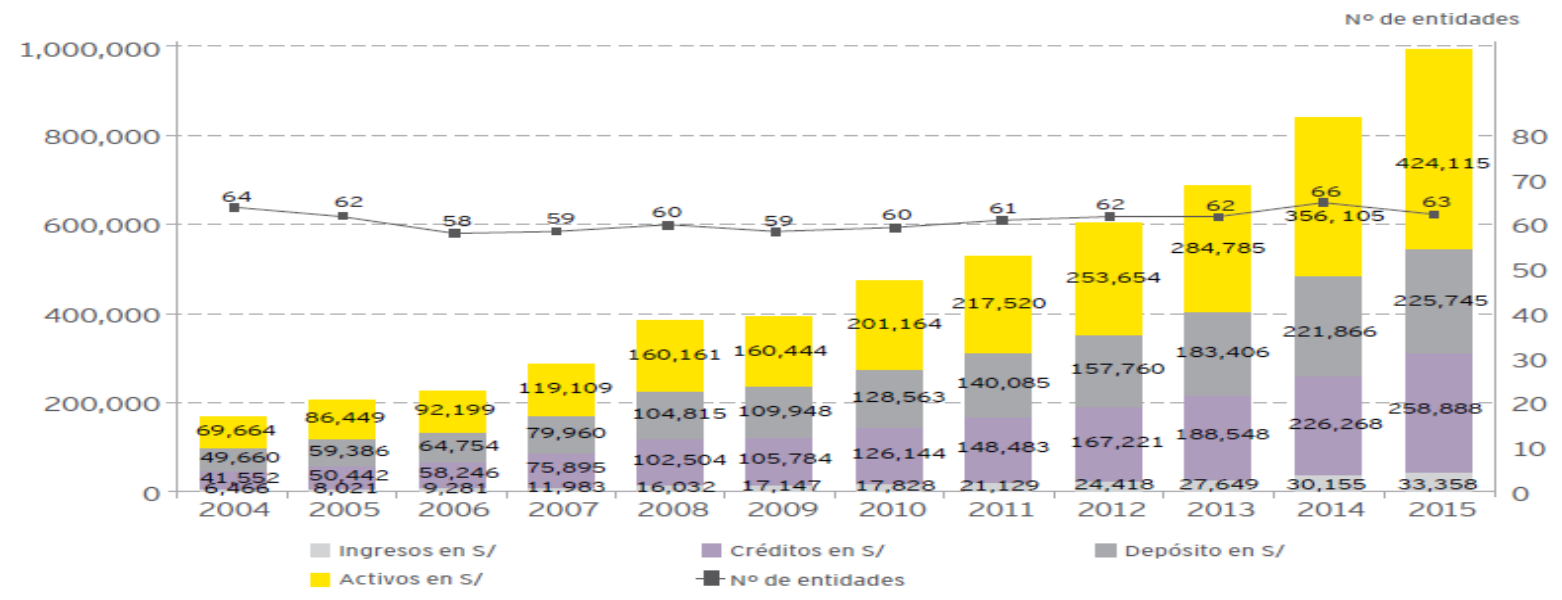

Fuente: Ey Perú (2016) 
La problemática se centra en la sede en Perú de una importante empresa multinacional de auditoría y consultoría, con presencia en más de 150 países, brindando servicios profesionales para las principales industrias del medio.

En el año 2015, contaba con más de 50 años de fundada y tenía más de 1000 colaboradores con sedes en Lima y provincias. Las áreas que se tenían eran: Auditoría, Impuestos, Transacciones y Finanzas corporativas y Consultoría.

En Consultoría se encontraba una sub área denominada consultoría para el sector financiero donde se enmarca el presente trabajo.

Esta sub área aún no estaba formalizada como tal, por lo cual no se tenía un proceso estructurado para reclutar y seleccionar practicantes y asistentes.

Debido al crecimiento del sector antes mencionado, esta sub área de consultoría tuvo un incremento de carga laboral, por ende se necesitó contratar una mayor cantidad de personas con un perfil especializado. El área de reclutamiento y selección vio necesario analizar el proceso y perfil de los contratados en la sub área para que puedan brindar un mejor servicio a sus clientes.

A su vez esta coyuntura venía acompañada por la escasez de talento que existía, que es la dificultad para encontrar candidatos idóneos especialmente de las carreras de ingeniería, la cual impedía satisfacer la demanda de contratación con profesionales especializados.

En la figura 3 se aprecia que Perú ocupa el segundo puesto a nivel mundial en dificultad de encontrar personal para cubrir las vacantes (Manpower Group, 2015). 
Figura 1.3.

Países con mayor dificultad para cubrir sus vacantes en el 2015

\section{JAPÓN, PERÚ y BRASIL fueron tres de los cinco países donde los empleadores reportaron mayor dificultad para cubrir sus vacantes también durante 2014 .}

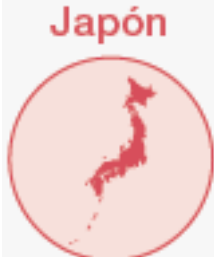

$83 \%$
Perú

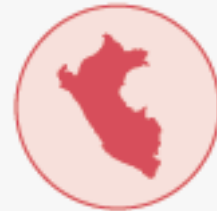

$68 \%$

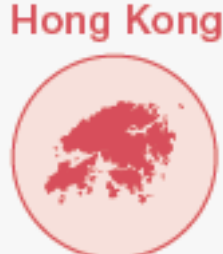

$65 \%$

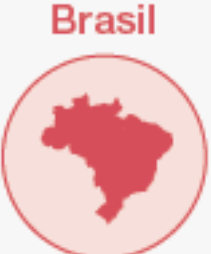

$61 \%$
Rumania

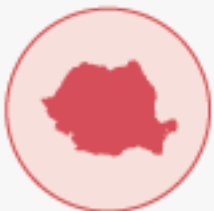

$61 \%$

Fuente: Manpower Group (2015)

Asimismo, en dicha encuesta menciona que el tercer puesto más difícil de cubrir, por cuarto año consecutivo es el de ingenieros.

Por el crecimiento económico antes mencionado, la cantidad de vacantes de practicantes y asistentes que necesitaba la sub área aumentó en 40\%. A raíz de esto, es que surge la necesidad de optimizar el proceso, la evaluación de candidatos, técnicas y desarrollo de acciones que permitan reclutar y seleccionar perfiles idóneos para la sub área.

Dentro de las principales problemáticas, se buscó solucionar la falta del flujo del proceso estableciendo tiempos para cada etapa, confirmación del perfil y competencias a evaluar. Además, no se tenía definida la estrategia de reclutamiento y acciones para conseguir los perfiles que se requerían, así como las técnicas de evaluación. Otra problemática era que el proceso era de dos meses, tiempo de cobertura extenso cuando en promedio para puestos iniciales en otras áreas era de un mes. Se tenían constantes reprogramaciones por la falta de disponibilidad de tiempo de los gerentes, lo que generaba malestar en los candidatos.

Lo expuesto nos lleva a plantearnos la siguiente pregunta: ¿Cómo se podrá lograr mayor efectividad en el proceso de reclutamiento y selección de practicantes y asistentes en la sub área de consultoría para el sector financiero de una empresa de servicios de auditoría y consultoría? 


\section{CAPÍTULO II: DESCRIPCIÓN DE LAS ACTIVIDADES Y TAREAS REALIZADAS}

En el 2015, se identifica la necesidad de realizar un plan de mejora para el proceso de reclutamiento y selección para la sub área de consultoría para el sector financiero considerando el incremento de contratación para la conformación de los equipos de proyectos. Por ende se realizaron dos procesos masivos para seleccionar a 20 practicantes y un proceso masivo para cubrir 10 posiciones de asistentes durante ese año.

El desarrollo de las actividades fueron supervisadas por la jefa de reclutamiento y selección, psicóloga titulada, en coordinación con los gerentes y los clientes internos. Fue un trabajo en equipo, conformado por los practicantes, asistentes, encargado y la jefa de reclutamiento.

A continuación se explicarán las actividades que se realizaron en el plan de mejora para este proceso:

\subsection{Elaboración el flujo del proceso de reclutamiento y selección}

Se inicia el despliegue del plan de mejora estableciendo el flujo del proceso que se desarrolló para atraer y seleccionar practicantes y asistentes. (Ver Apéndice 1) Aquí se establecieron las etapas y tiempo promedio para cada una de ellas. Cabe señalar que la implementación de mejora fue en los siguientes puntos.

\subsubsection{Precisión del perfil}

El primer paso fundamental dentro del flujo del proceso de reclutamiento y selección es el análisis del descriptivo del puesto para la elaboración del perfil. Según Alles (2015) "una correcta definición de aquello que se desea encontrar será el primer paso para el éxito en un proceso de selección de personas. Las descripciones de puestos son la base de un buen sistema de gestión de Recursos Humanos” (p. 156). 
Para el levantamiento de la información, se entrevistó a los gerentes para comprender el negocio, contexto donde realizarán sus tareas, formación, requerimientos y conocimientos específicos y línea de carrera. Con ello se elaboraron los perfiles para ambos puestos. Esto permitió realizar una búsqueda más aterrizada y clara, con la finalidad de disminuir el tiempo del proceso. En base al diccionario de competencias de la empresa, se seleccionaron las competencias y comportamientos, en conjunto con la sub área solicitante, interviniendo el área de reclutamiento como asesor en esta elección. Esto iba a permitir a los entrevistadores evaluar con mayor exactitud. Alles (2015) señala que "la selección por competencias, a través de describir lo requerido para un desempeño exitoso en un determinado puesto de trabajo, abriéndolo en una serie de ítems (competencias), permite una mejor evaluación de los posibles candidatos." (p. 46). La información recopilada se plasmó en el formato del perfil que se manejaba dentro de la empresa, el cual fue revisado y aprobado por la jefa de reclutamiento y selección y el gerente principal de la sub área encargada.

\subsubsection{Identificación de la estrategia de reclutamiento y acciones de atracción}

Se vio conveniente realizar reclutamiento externo para practicantes y mixto (interno y externo) para asistentes. No se había explorado anteriormente esta alternativa para la sub área y había colaboradores con buena evaluación de desempeño e interesados en explorar nuevas oportunidades de crecimiento. Chiavenato (2009) indica que "el reclutamiento interno y el externo contribuyen a la formación y la continua actualización del banco de talentos que servirá de fuente para los reclutamientos futuros" (p. 117). 
La propuesta de valor para el reclutamiento externo era demostrar a la empresa como una institución multinacional, que impulsa el aprendizaje continuo, la experiencia, orientación y las oportunidades de crecimiento. Esto es valorado por la generación "millennial”, que era el público objetivo a seleccionar. Ante esto se requería difundir las convocatorias por los canales digitales mostrándoles las oportunidades de aprendizaje y crecimiento que se ofrece.

Para ello se realizaron las siguientes acciones: convocatoria interna, publicaciones en la red social de Facebook de la empresa, publicaciones en bolsas de trabajo de universidades, en el Ministerio de trabajo y en la bolsa laboral de Bumeran, difusión en los grupos de redes sociales de los centros estudiantiles en el caso de las universidades nacionales y campaña de referidos que se lanzó en el área de consultoría.

En el caso de las coberturas de asistentes de las carreras de ingeniería, se realizaron búsquedas proactivas y armado de base de datos utilizando la fuente LinkedIn. También se hicieron publicaciones en Bumeran para tener “bolsas de curriculums” que se podrían necesitar a lo largo del año.

Se eligieron a dos gerentes de la sub área para realizar la validación de curriculums pre filtrados.

\subsubsection{Establecimiento de las técnicas de evaluación}

Se incorporó el assessment center como metodología de evaluación para la selección de practicantes y asistentes. Luego se continuaría con la etapa de pruebas psicológicas, entrevistas por competencias con el área de reclutamiento y selección y con los gerentes de la sub área. Alles (2015) 
define al Assessment center como "pruebas situacionales donde se enfrenta a los candidatos con la resolución práctica de situaciones conflictivas reales del entorno del puesto del trabajo, considerado como una buena práctica de Recursos Humanos" (p. 308). Porret (2014) indica que "la actuación por parte del Assessment center comporta un profundo conocimiento de la empresa, de los contenidos de los puestos de trabajo y de las capacidades requeridas para su desempeño puesto que se realizan las pruebas a medida de cada organización" (p. 165).

Se utilizó un caso de negocio de la plataforma de reclutamiento de la empresa a nivel global y fue adaptado por dos gerentes y la jefa del área de reclutamiento. Se solicitó la revisión a otros jefes y asistentes de la misma sub área lo que llevó a ciertas modificaciones y colocar un glosario de términos para que quede claro para los participantes. En él se presentaba la problemática de una empresa del rubro financiero. Se evaluaba cómo analizaban y llegaban a tomar decisiones en equipo frente al problema planteado. Se elaboró una guía para los clientes internos donde se especificaban las competencias y comportamientos. Esto con la finalidad de que la evaluación de los candidatos sea más objetiva (Ver Apéndice 2).

La evaluación se realizaba los sábados en la mañana donde había una presentación y luego los candidatos iban a las salas de reuniones a desarrollar el caso. Se eligió los sábados debido a que ese día la tasa de asistencia era mucho mayor y los gerentes de área contaban con disponibilidad. (Ver Apéndice 3)

Los evaluadores eran los miembros del equipo de reclutamiento, Business Partners de recursos humanos y los gerentes (clientes internos). Se 
realizaban reuniones previas para el estudio del caso y la aplicación. Eran dos por cada equipo de máximo ocho candidatos. Se dividía en dos etapas: la primera denominada "Desarrollo del caso" donde analizaban la información, debatían las problemáticas y posibles soluciones para la decisión final. La segunda etapa denominada "Exposición del caso" sustentaban las soluciones elegidas y se intercambiaba los dos evaluadores previos. Con esto se buscaba contar con cuatro opiniones, dos que evaluaron las competencias en la etapa de "Desarrollo del caso" y dos que evaluaron las competencias en la “Exposición del caso", en todos los casos la evaluación es del 1 al 5, donde 1 es insatisfactorio y 5 es excelente. Al final de la jornada, se realizaban mesas de calibración con las evaluaciones, observaciones y comentarios obtenidos. Debían obtener en promedio calificación 3 para pasar a la siguiente fase. A los seleccionados se les enviaba las pruebas online por medio de "Evaluar.com" que arrojaba el resultado de las pruebas y ahorraba tiempo en la aplicación y corrección de las mismas. Se enviaban las siguientes pruebas psicológicas: "Test de Dominós” que evalúa inteligencia no verbal y el "16 PF” que evalúa personalidad. Los resultados de las pruebas eran contrastados en las entrevistas por competencias. La metodología utilizada era la denominada estrella (S T A R), donde se le pregunta al candidato que comente sobre una experiencia laboral previa (situación) e indique en qué consistió la tarea, la acción que realizó y qué resultados obtuvo. Se evaluaba también la motivación y que tuvieran valores alineados a la empresa. Pereda, Berrocal y Alonso (2011) explican que "la entrevista de competencias surge como respuesta ante las numerosas críticas y objeciones a la subjetividad y poca fiabilidad de la entrevista clásica, ya que permite combinar sus 
características con las de los procedimientos estandarizados de evaluación de personal" (p. 69). Si el candidato aprobaba las etapas anteriormente mencionadas, continuaban a la entrevista con el gerente que evaluaba los conocimientos técnicos.

\subsubsection{Elaboración de referencias laborales}

Las referencias laborales consisten en solicitar información sobre el desempeño que tuvo el candidato en sus anteriores centros de trabajo. En un inicio no se solicitaban y se implementó para las posiciones de asistentes. Según Gomez-Mejía, Balkin, Cardy (2008), indican que "uno de los mejores métodos para predecir el éxito futuro de los posibles empleados consiste en comprobar su historial laboral anterior" (p. 212).

\subsubsection{Preparación de informe}

Se incorporó en el proceso la preparación de informes de los candidatos. Esto con un reporte visualmente atractivo y conciso que era lo que solicitaba la sub área. (Ver Apéndice 4) Los informes eran revisados por la jefa y encargada de reclutamiento.

\subsubsection{Cierre del proceso}

En base a las ponderaciones de las evaluaciones se realizaban las entrevistas de cierre a los candidatos seleccionados. A los que no habían sido seleccionados se les enviaba un correo de agradecimiento donde se les indicaba que su información quedaría guardada en la base de datos para futuras oportunidades laborales.

Al mes de ingreso del personal contratado, se aplicaron encuestas para contar con retroalimentación sobre el proceso. (Ver Apéndice 5) 


\section{CAPÍTULO III: RESULTADOS DE LA INTERVENCIÓN}

A continuación, se exponen los resultados obtenidos por el plan de mejora para el proceso de reclutamiento y selección para la sub área de consultoría para el sector financiero.

Con la precisión del perfil se estableció que se necesitarían practicantes pertenecientes a tercio, quinto, décimo superior que cuenten con mínimo seis meses de experiencia preprofesional de escuelas de negocios e ingeniería. Para asistentes debían contar con mínimo un año de experiencia, sin considerar prácticas de las carreras de ingeniería industrial, de sistemas, estadísticas o afines y contar con ciertos conocimientos técnicos que se requería para ese sector.

Las competencias seleccionadas para practicantes y asistentes fueron: capacidad de análisis, trabajo en equipo, comunicación eficaz y capacidad de planificación y organización. (Ver Apéndice 2). Se detallaron los comportamientos de acuerdo al diccionario de la empresa y fue la guía en la evaluación del assessment.

Sobre las acciones desarrolladas para atraer a los candidatos, se puede observar en la siguiente figura que los medios de mayor efectividad fueron Facebook de la empresa y bolsas de universidades.

Figura 3.1.

Medios de reclutamiento utilizados

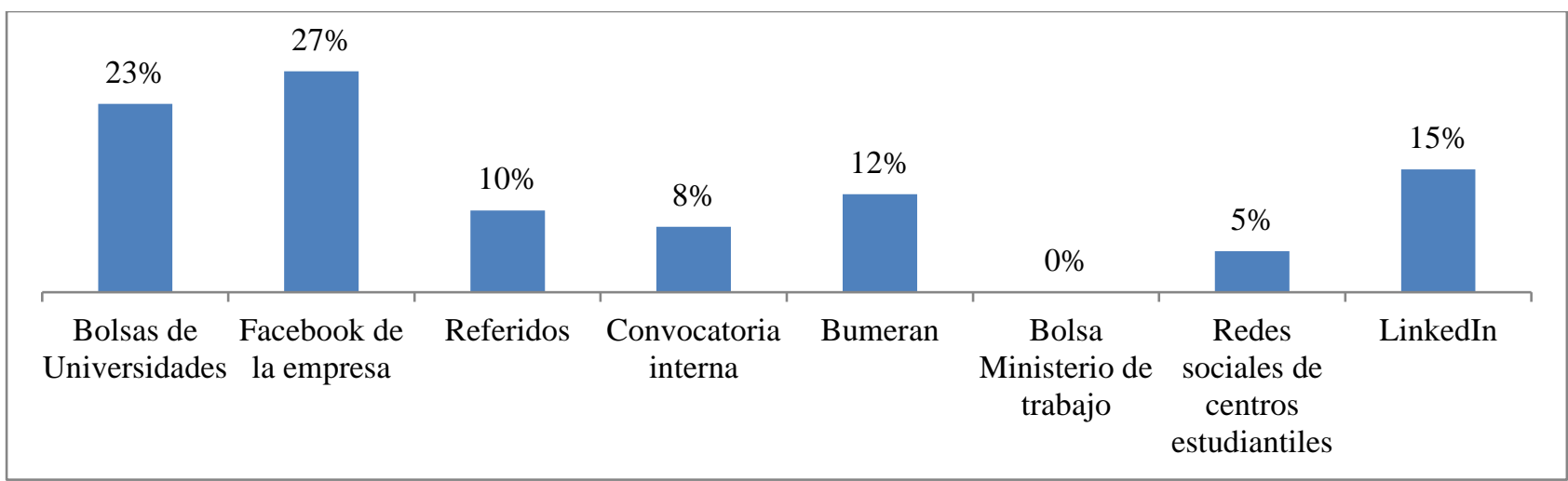

Fuente: Empresa de auditoría y consultoría, 2015. Elaboración propia 
Al aumentar en un 40 por ciento la demanda de posiciones para la sub área de consultoría para el sector financiero, se realizaron dos procesos masivos para cubrir las 20 vacantes de practicantes y un proceso masivo para cubrir las 10 vacantes de asistentes con el objetivo de poder ampliar el universo de postulaciones, donde se obtuvieron los resultados presentados en las tablas 1 y 2.

Tabla 3.1.

Resultados de los dos procesos masivos de practicantes

\begin{tabular}{ccc}
\hline Etapas del proceso de selección & Estado del candidato & Número de candidatos \\
\hline & Convocados & 160 \\
Assessment center & Participantes & 140 \\
& Aprobados & 60 \\
\hline Pruebas psicológicas y entrevistas por & Participantes & 60 \\
competencias con Recursos Humanos & Aprobados & 40 \\
\hline Entrevistas con Gerentes de la & Participantes & 40 \\
sub área & Aprobados & 20 \\
\hline Cierre & Contratados & 20 \\
\hline
\end{tabular}

Fuente: Empresa de auditoría y consultoría. Elaboración propia

Tabla 3.2.

Resultados del proceso masivo de asistentes

\begin{tabular}{ccc}
\hline Etapas del proceso de selección & Estado del candidato & Número de candidatos \\
\hline & Convocados & 80 \\
Assessment center & Participantes & 60 \\
& Aprobados & 40 \\
\hline $\begin{array}{c}\text { Pruebas psicológicas y entrevistas } \\
\text { por competencias con Recursos } \\
\text { Humanos }\end{array}$ & Participantes & 30 \\
\hline Entrevistas con Gerentes de la & Aprobados & 20 \\
sub área & Participantes & 20 \\
\hline Entrevistas con Gerentes de la & Aprobados & 10 \\
sub área & Contratados & 10 \\
\hline Cierre &
\end{tabular}

Fuente: Empresa de auditoría y consultoría. Elaboración propia

Siguiendo el proceso establecido se detalla el impacto que tuvo en los indicadores de negocio de la empresa: 
- Se logró cubrir al 100 por ciento las posiciones de practicantes y asistentes, posiciones solicitadas en el año 2015.

- El proceso influyó de manera positiva en la rentabilidad de la empresa por dos motivos:

- Al disminuir el tiempo del proceso de selección de practicantes y asistentes de dos meses a tres semanas, disminución del 63 por ciento, se podía tener antes al personal disponible para cumplir las funciones establecidas.

- Al conseguir un personal especializado y/o con experiencia específica hace que la curva de aprendizaje del contratado sea menor, lo cual contribuye a maximizar el valor que éste brinda a la institución.

Por otro lado, se realizaron encuestas al personal contratado con el nuevo proceso. De los 30 ingresantes se obtuvieron 20 respuestas y como principales puntos se obtuvo que:

- El medio más efectivo en la difusión de la convocatoria fue el Facebook de la empresa con 30 por ciento y bolsas de trabajo de universidades con 20 por ciento.

- Los medios más utilizados por los postulantes al momento de buscar ofertas laborales son Facebook con 35 por ciento y bolsas de las universidades con 20 por ciento.

- El tiempo del proceso de selección les pareció bueno a un 53 por ciento y excelente a un 18 por ciento.

- El proceso de selección en términos generales les pareció muy bueno a un 43 por ciento y excelente a un 30 por ciento.

El detalle de los demás resultados de la encuesta se pueden observar en el Apéndice 5.

En resumen, las actividades implementadas tuvieron buena aceptación por parte de la sub área y se obtuvo óptimos resultados como los anteriormente mencionados. Asimismo, se pudo implementar la metodología del Assessment center para posiciones de practicantes y asistentes en otras áreas obteniendo mejoras en el tiempo de los procesos. 


\section{CONCLUSIONES}

- Las mejoras implementadas en el proceso de reclutamiento y selección para practicantes y asistentes originaron un impacto positivo en la rentabilidad de la empresa por la disminución del tiempo del proceso y por la incorporación de profesionales especializados para los proyectos de la sub área.

- En la elaboración del perfil del candidato es fundamental realizar el levantamiento de información cada vez que se inicia un proceso de selección con la finalidad de tener claras las características de lo que se busca y el entorno donde trabajarán debido a que los requisitos específicos pueden variar. Es importante la retroalimentación del área solicitante del puesto en esta etapa.

- Al establecer los medios para la difusión de las convocatorias, se debe tener en cuenta cuál es nuestro público objetivo para utilizar los más adecuados.

- Realizar búsquedas proactivas en LinkedIn y contar con una base de datos para futuras búsquedas contribuye a acelerar el proceso de reclutamiento y selección.

- La técnica de evaluación denominada assessment center puede ser utilizado para puestos iniciales como practicantes y asistentes cuando se desea seleccionar a un mayor número de candidatos en menor tiempo.

- Las referencias laborales sirve para verificar el desempeño pasado del candidato el cual es importante realizar dentro del proceso de selección. 


\section{RECOMENDACIONES}

- Si se va a utilizar un caso de negocios para realizar un assessment center, se recomienda que sea probado en un grupo piloto con participantes similares a los cuales se les aplicará en la selección esto con la finalidad de incorporar mejoras en la aplicación real.

- Se recomienda analizar los medios más efectivos que se usarán al momento de hacer la difusión de la convocatoria de acuerdo al público objetivo que se desea atraer con la finalidad de utilizar los más adecuados.

- Para la difusión de las convocatorias, puede revisarse la utilidad de otros medios digitales como por ejemplo LinkedIn, Instagram, Twitter, Youtube, entre otros, de acuerdo a los perfiles que se busquen.

- Es importante establecer una revisión constante del proceso para realizar mejoras en él. Se recomienda revisar plataformas más completas de selección que se encarguen de automatizar y simplificar las etapas operativas para contar con la información centralizada del proceso para una rápida y fácil extracción.

- Se pueden elaborar encuestas para los clientes internos para conocer la satisfacción en relación al proceso de selección desarrollado para contar con información para mejoras en el mismo. 


\section{REFERENCIAS}

Alles, M. (2012). Selección por competencias (5ª ed.). Buenos Aires: Granica.

Alles, M. (2015). Dirección Estratégica de Recursos Humanos. Gestión por competencias (3 ${ }^{a}$ ed.). Buenos Aires: Granica.

Chiavenato, I. (2009). Gestión del talento (3 ${ }^{\mathrm{a}}$ ed.). México D.F.: McGraw-Hill.

EY Perú (2016). Guía de negocios e inversión en el Perú. Lima: Ey Perú Library.

Gómez-Mejía, L., Balkin, D. y Cardy, R. (2008). Gestión de Recursos Humanos (5ª ed.). Madrid: Pearson Prentice Hall.

Pereda, S., Berrocal, F. y Alonso, M. (2011). Técnicas de gestión de recursos humanos por competencias ( $3^{\mathrm{a}}$ ed.). Madrid: Editorial Universitaria Ramón Areces.

Porret, M. (2014). Gestión de personas: Manual para la gestión del capital humano en las organizaciones ( $6^{\mathrm{a}}$ ed.). Madrid: ESIC Editorial.

Salgado, J. F. y Moscoso, S. (2008). Selección de Personal en la Empresa y las AAPP: De la visión tradicional a la visión estratégica. Papeles del Psicólogo, 29(1), 16-24.

Manpower Group. (2015). Encuesta de escala de talento. Recuperado de https://www.manpower.com.pe/mpintranet/publicaciones/3166-9795432090759.pdf

Instituto Nacional de Estadística e Informática (2018). Producción nacional. Recuperado de https://www.inei.gob.pe/media/principales_indicadores/informe-tecnico-deproduccion-nacional-febrero2019.PDF 


\section{APÉNDICES}




\section{APÉNDICE 1: Flujo del proceso de reclutamiento y selección}

Figura 5. Flujo del proceso de reclutamiento y selección de la Empresa de auditoría y consultoría. Elaboración propia

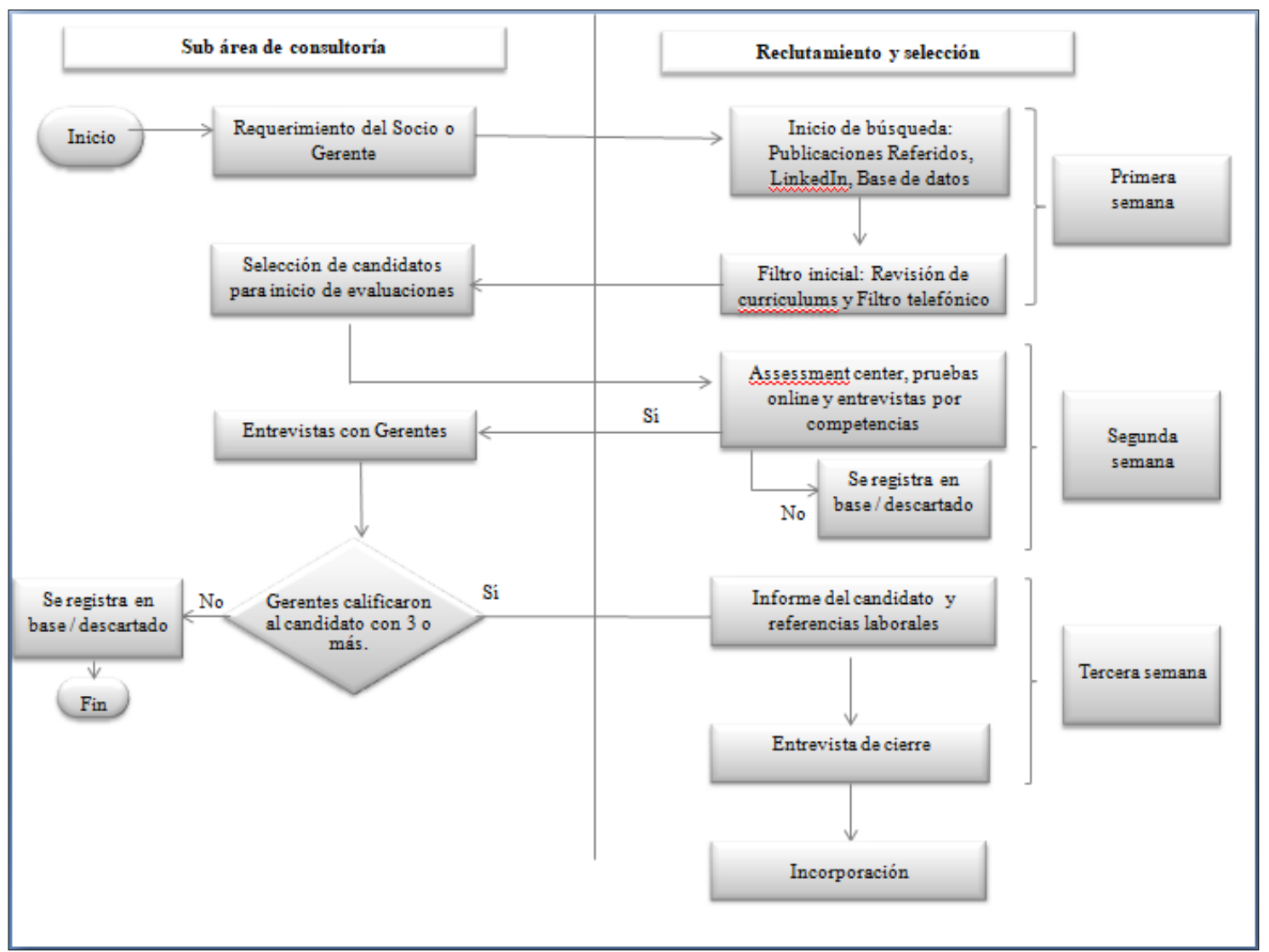




\section{APÉNDICE 2: Guía de evaluación para el Assessment center}

Figura 6. Guía de evaluación para el assessment center. Elaboración propia

Desarrollo del caso

Ficha de evaluación

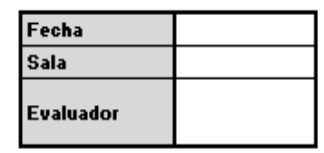

\begin{tabular}{|c|c|c|c|c|c|c|}
\hline Candidato & $\begin{array}{c}\text { Capacidad de } \\
\text { ańlisis de } \\
\text { problemas }\end{array}$ & $\begin{array}{l}\text { Capacidad de } \\
\text { planificación } \\
\text { organización }\end{array}$ & $\begin{array}{c}\text { Comunicación } \\
\text { Efectiva }\end{array}$ & $\begin{array}{c}\text { Trabajo en } \\
\text { equipo }\end{array}$ & Promedio & $\begin{array}{l}\text { Observaciones } \\
\text { IComentarios }\end{array}$ \\
\hline 1 Candidato 1 & & & & & & \\
\hline 2 Candidato 2 & & & & & & \\
\hline 3 Candidato 3 & & & & & & \\
\hline 4 Candidato 4 & & & & & & \\
\hline 5 Candidato 5 & & & & & & \\
\hline 6 Candidato 6 & & & & & & \\
\hline 7 Candidato 7 & & & & & & \\
\hline 8 Candidato 8 & & & & & & \\
\hline
\end{tabular}

Lenenda para la evaluación: Seleccione una de las calific aciones de la escala para evaluar la competencia. 5 E Excelente: el candidato posee un nivel extraordinariamente alto de competenciasthabilildades

4= Muy bueno: el candidato posee un nivel alto de competenciasthabilidades

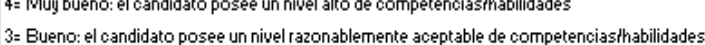

2= Regular: el candidato presenta algunas deficiencias en cuanto a las competenciasthabilidades relacionadas con el trabajo

I= Insatisfactorio: El candidato presenta deficiencias significativas en las competenciasthabilidades relacionadas con el trabajo NHÁ = competencia no aplicable ono evaluada

\begin{tabular}{|c|c|}
\hline & Guía de evaluación \\
\hline $\begin{array}{l}\text { Capacidad de } \\
\text { análisis de } \\
\text { problemas }\end{array}$ & 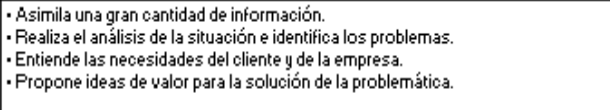 \\
\hline $\begin{array}{c}\text { Capacidad de } \\
\text { planificación y } \\
\text { organización }\end{array}$ & 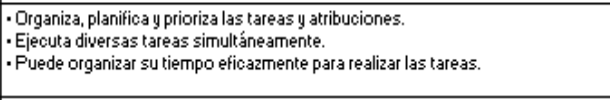 \\
\hline $\begin{array}{l}\text { Comunicación } \\
\text { efectiva }\end{array}$ & 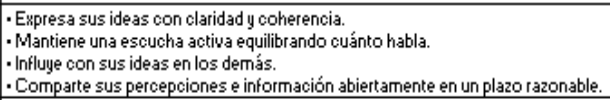 \\
\hline Trabajo en equipo & \begin{tabular}{|l} 
- Colabora con los demás para lograr el objetivo en común. \\
- Promueve el intercambio de ideas respeltando las opiniones de los demás. \\
- Demuestra compromiso y responsabilidad con una visión compartida.
\end{tabular} \\
\hline
\end{tabular}




\section{APÉNDICE 3: Cronograma del proceso masivo para la selección de practicantes y asistentes}

Primera semana del proceso de selección

\section{1ra Etapa - Programa del proceso masivo}

8:15 a.m. Reunión de coordinación

8:45 a.m. Proyección de videos sobre la cultura de la empresa conforme van llegando los participantes.

9:00 a.m. Bienvenida y Presentación

9:20 a.m. Testimonios de crecimiento de colaboradores

9:35 a.m. Indicaciones del ejercicio a realizar

9:40 a.m. Inicio de actividad en salas de reunión (revisión del caso)

- Lectura individual del caso (10 minutos)

- Discusión guiada (20 minutos)

- Desarrollo (50 minutos)

11:00 a.m. Coffee break/Interacción candidatos y gerentes.

11:30 a.m. Exposiciones grupales

12:30 p.m. Cierre de jornada

\section{Segunda semana - Evaluaciones y Entrevista RRHH}

Se enviaban las evaluaciones online y se realizaban las entrevistas por competencias a cargo del área de Recursos Humanos.

\section{Tercera semana - Entrevistas con el área y entrevistas de cierre}

Se realizaban las entrevistas con los gerentes y si aprobaban su contratación, inmediatamente después se realizaban las entrevistas de cierre con los candidatos seleccionados. 


\section{APÉNDICE 4: Modelo de informe del candidato}

Figura 7. Modelo de informe del candidato. Elaboración propia

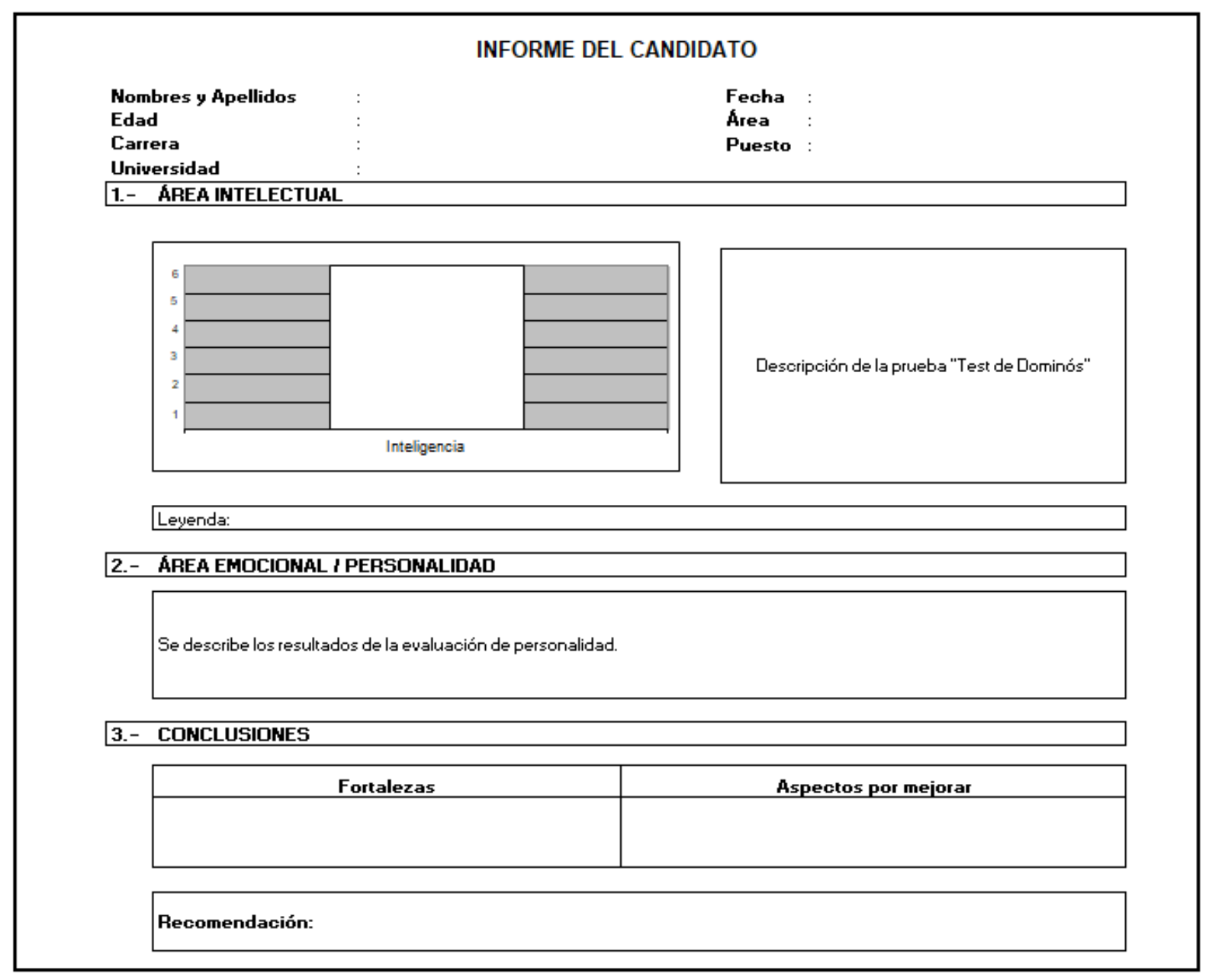




\section{APÉNDICE 5: Encuesta realizada a los nuevos ingresos por la metodología de Assessment center}

Figura 8. Encuesta realizada a los nuevos ingresos por asessment center. Elaboración propia

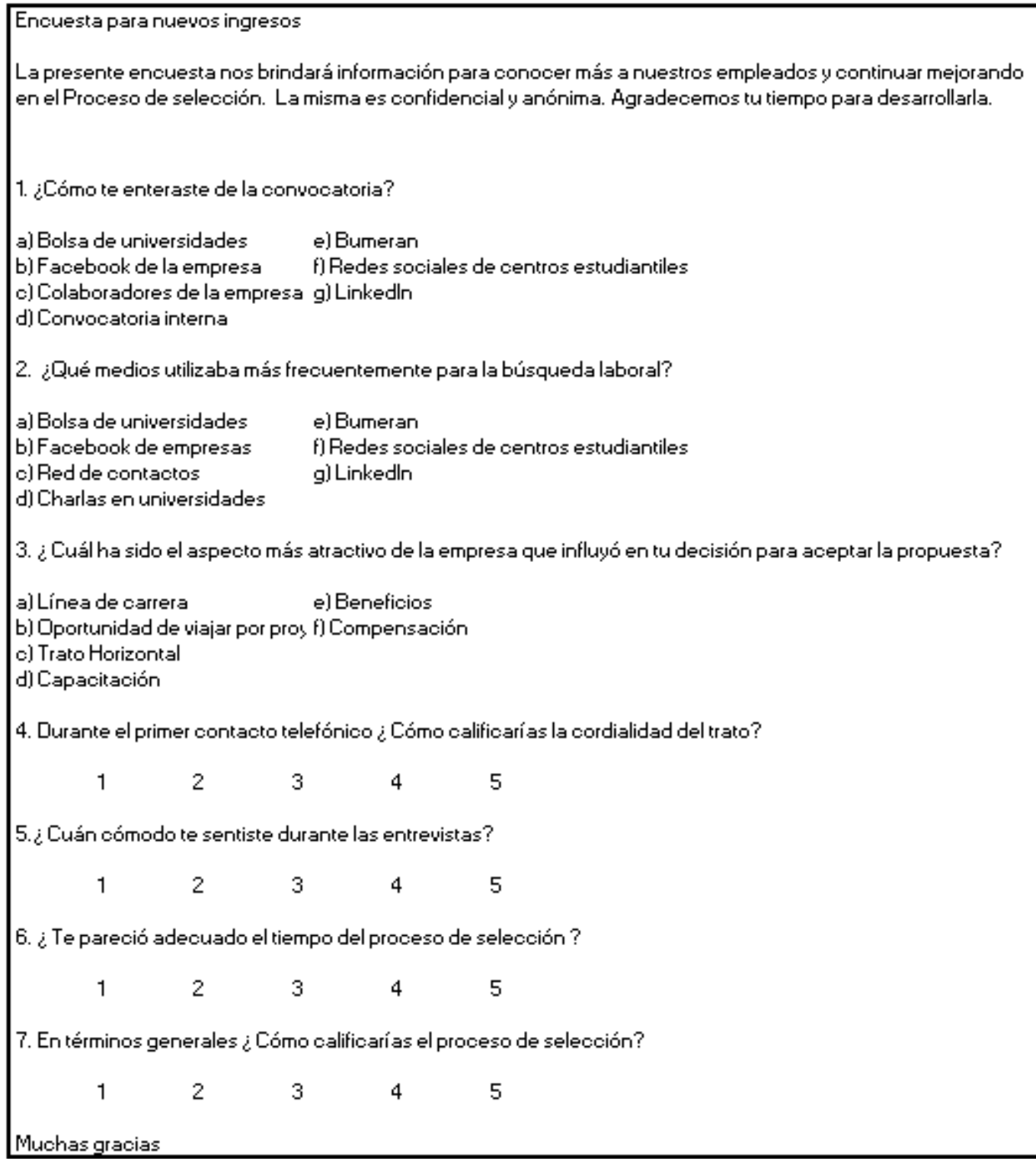


Figura 9. Resultados pregunta 1. Encuesta a colaboradores de empresa de auditoría y consultoría.

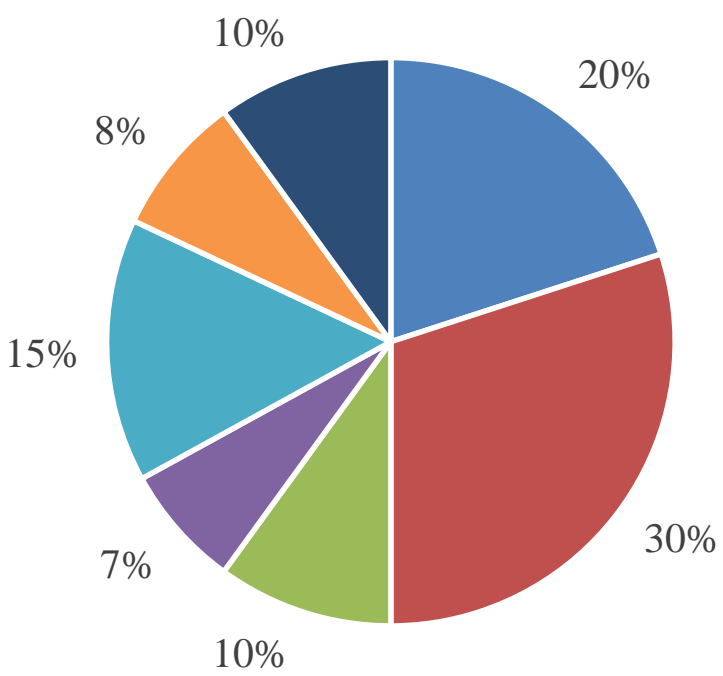

- Bolsa de universidades

- Facebook de la empresa

- Colaboradores de la empresa

- Convocatoria interna

- Bumeran

- Redes sociales de centros estudiantiles

Figura 10. Resultados pregunta 2. Encuesta a colaboradores de empresa de auditoría y consultoría.

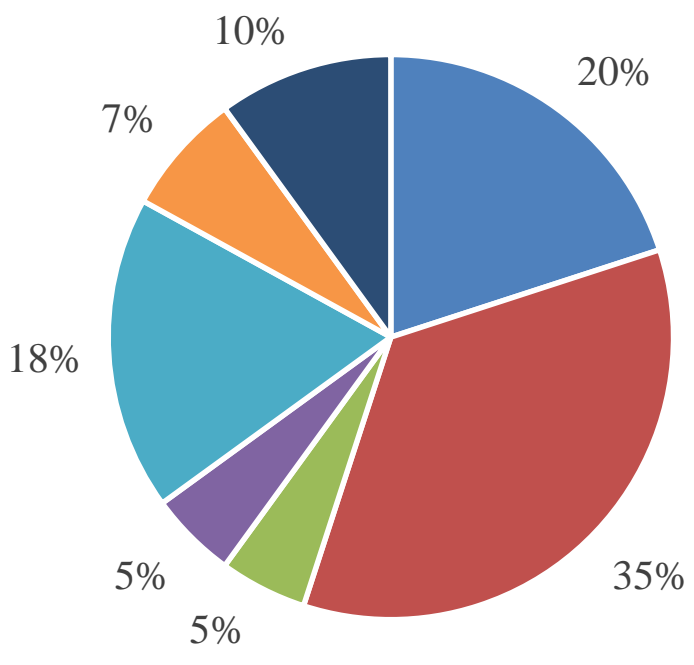

- Bolsa de universidades

- Facebook de empresas

- Red de contactos

- Charlas en universidades

- Bumeran

- Redes sociales de centros estudiantiles 
Figura 11. Resultados pregunta 3. Encuesta a colaboradores de empresa de auditoría y consultoría.

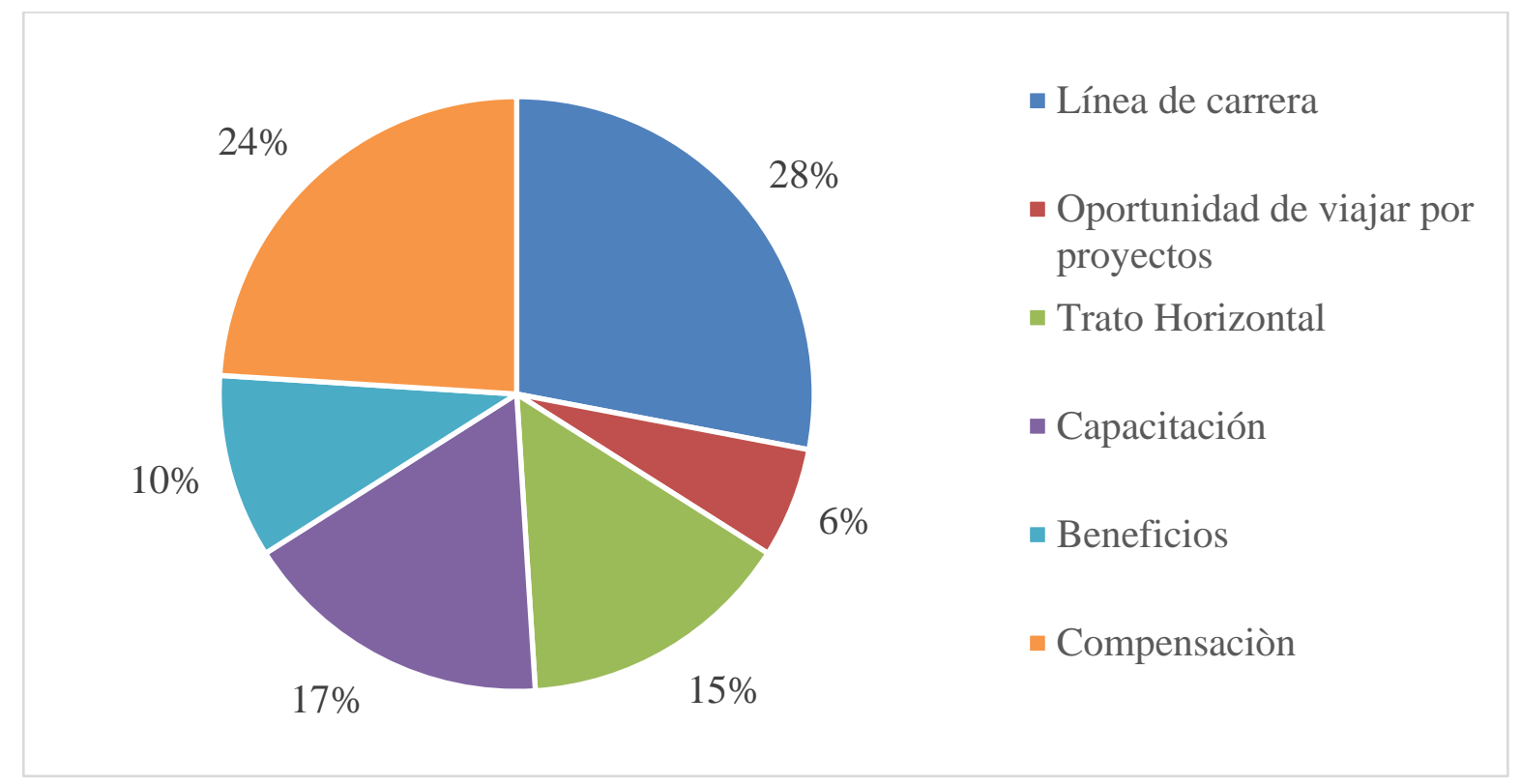

Figura 12. Resultados pregunta 4. Encuesta a colaboradores de empresa de auditoría y consultoría.

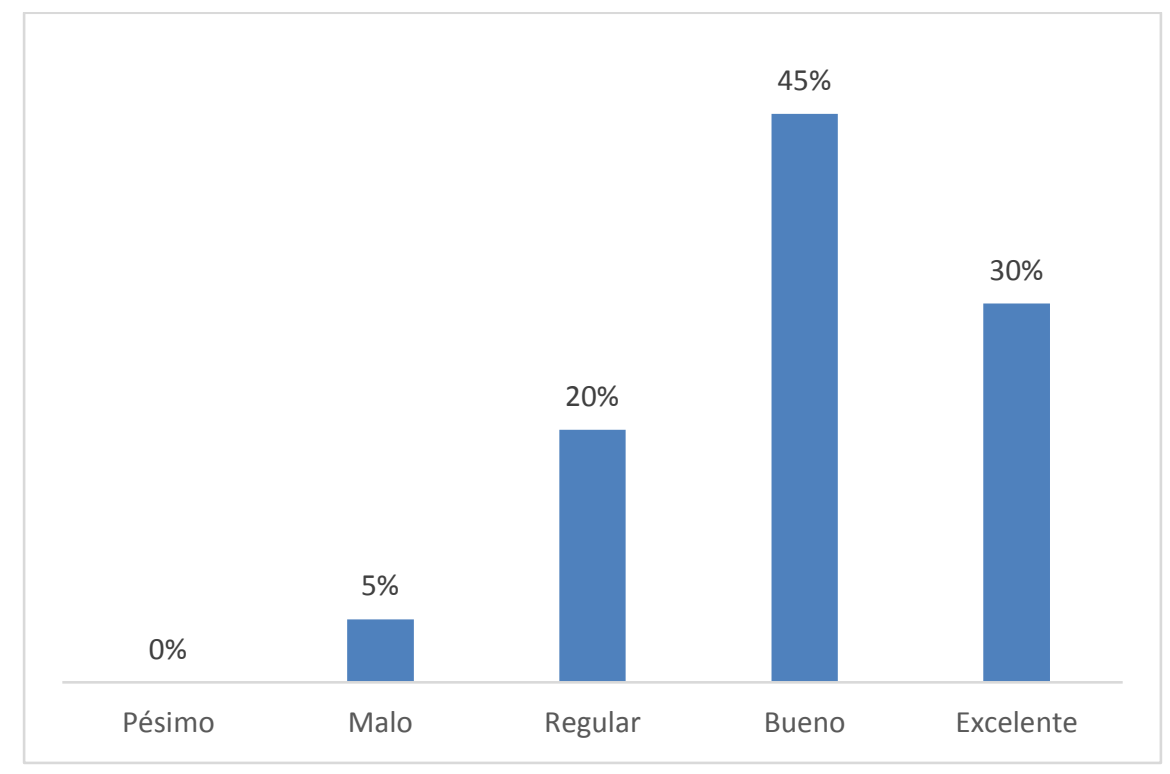


Figura 13. Resultados pregunta 5. Encuesta a colaboradores de empresa de auditoría y consultoría.

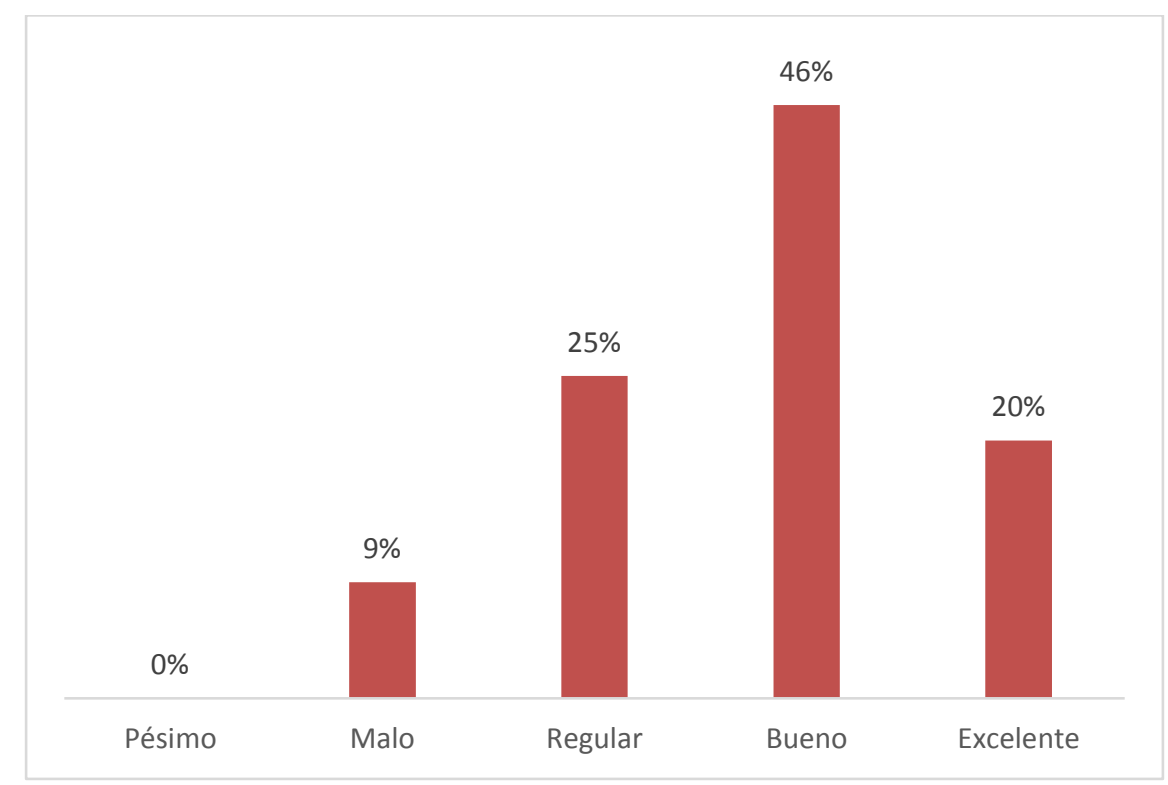

Figura 14. Resultados pregunta 6. Encuesta a colaboradores de empresa de auditoría y consultoría.

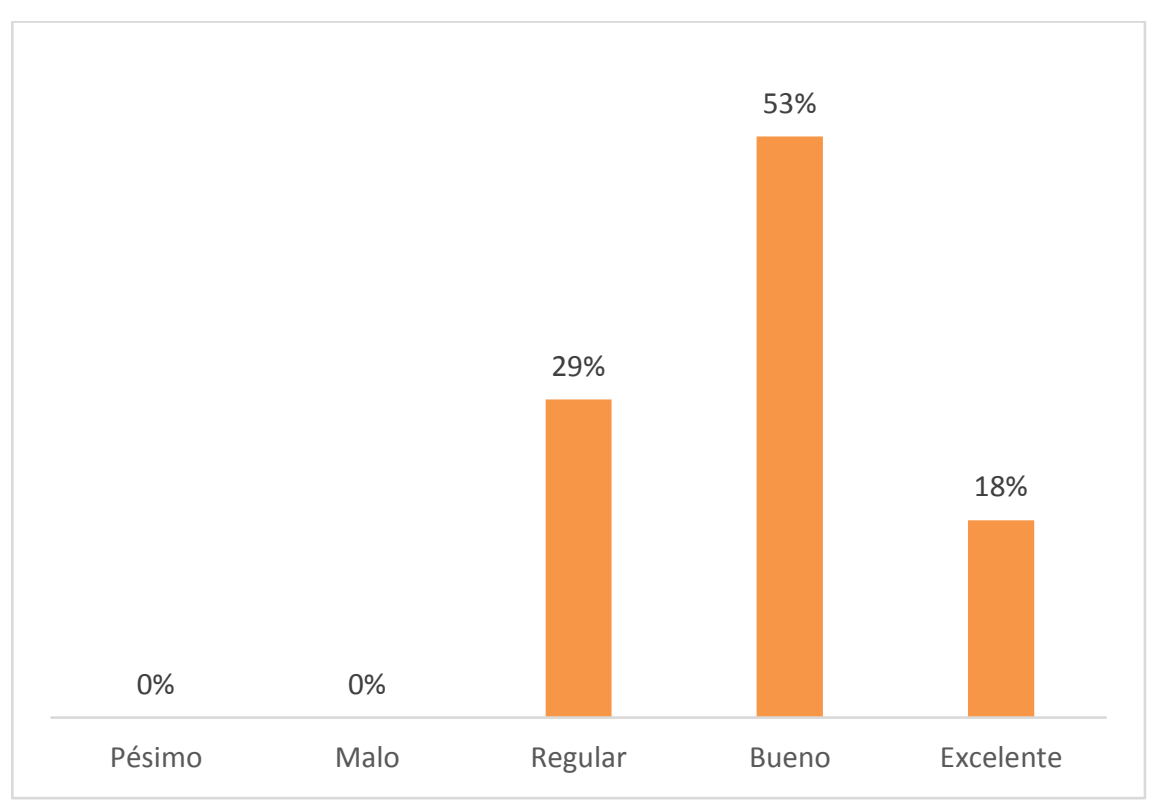


Figura 15: Resultados pregunta 7. Encuesta a colaboradores de empresa de auditoría y consultoría.

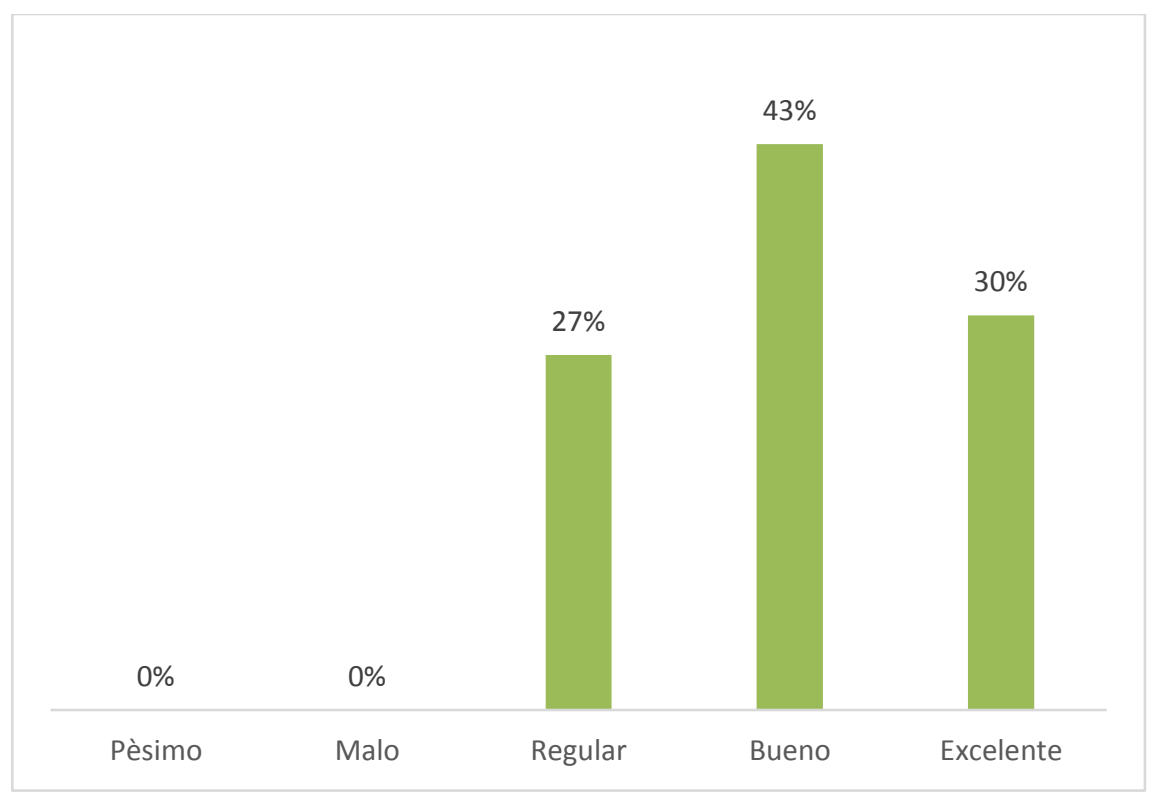

